\title{
An Effective Grayscale Conversion with Applications to Image Enhancement
}

\author{
Codruta O. Ancuti \\ Cosmin Ancuti \\ Philippe Bekaert \\ Hasselt University, Belgium, firstname.lastname@uhasselt.be
}
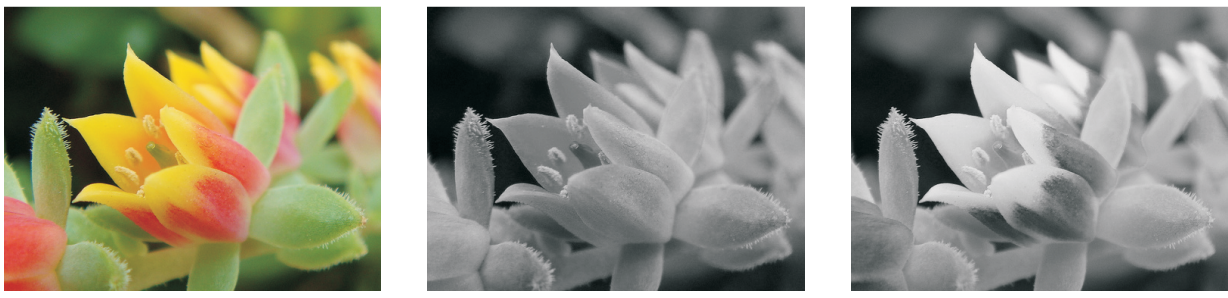

Figure 1: From left to right: color image, result of standard grayscale transformation and our decolorized image.

Introduction. Recent efforts to accurately integrate properties of human visual perception have fueled an impressive improvement not only in digital photography but also in color manipulation tools. Color is one of the main attributes that influences human visual system, being ultimately the result of our experience and perception. However, several important applications such as substitution systems for visually impaired people, computerized tomography, printed textbooks and aesthetical stylization still require a reliable grayscale representation of images. Mapping three dimensional color information onto a single dimension while still preserving the original appearance, contrast and finest details is not a trivial task. Standard monochromatic transformations, found in commercial image editing software, neglect the color distribution, being unable to conserve in many cases the discriminability of the original chromatic contrast. Consequently, isoluminant colors are mapped to similar grayscale levels increasing the ambiguity in the decolorized image version. Although luminance is a definite value, our subjective interpretation that measures apparent brightness is very important in image decolorization process.

This problem has been addressed recently by several approaches [Gooch et al. 2005; Grundland and Dodgson 2007; Smith et al. 2008]. Apart from solving the discriminability of the compressed chromatic levels, for a perceptually accurate and efficient conversion other characteristics are very important too. In general, due to quantization strategies or prohibitive function optimization, many of the existing approaches fail to render the original image look, to preserve the finest details and luminance consistency (shadows and highlights should not be reversed). Additionally, a crucial problem is the computational efficiency for real time applications. We argue that the concept of image decolorization is not to obtain a perfect optical match but rather to obtain a plausible image that maintain the overall appearance and the most salient features, which improves the discriminability of the scenes objects.

Our Approach. This paper introduces an alternative decolorization approach. In our scheme the luminance level is progressively augmented by the chromatic variation of the salient information. Saliency, a well studied topic in computer vision, aims to emulate the attention mechanism of human visual system. Our technique maps dependently by the prominent chromatic information the global lightness embracing the color opponency theory [Judd 1966], generally accepted as a foundation of many visual saliency models [Itti et al. 1998]. Color opponency theory has been successfully applied also in related areas such as color harmonization, detail enhancement and tone management. After the monochromatic luminance channel is filtered and stored as a reference, the luminance values are computed pixel-wise by mixing both saturation and hue values that creates a new spatial distribution with the consequence of increasing the contrast of the interest regions. All the pre-computed values are normalized in order to fit the gray shades range. The intensity is re-balanced in order to conserve the amount of glare in the initial image. For extreme lighting conditions we apply several constraints to avoid clipping and fading of the apparent details.

The method has been verified for a large database of color images, including the standard examples commonly used to test grayscale techniques. To validate our technique a comprehensive comparison but also a perceptual evaluation against the existing state-ofthe-art methods have been performed (the reader is referred to the additional material). Our approach, although conceptually straightforward, is able to decolorize images competitively with the more elaborate techniques. The runtime is linear, being suitable for realtime applications (e.g. the method of [Gooch et al. 2005] needs 25.7 seconds for a $200 \times 200$ image - GPU implementation). Even though a small set of parameters allow the users to control the grayscale conversion, the method still performs well with default parameters. Since our decolorization strategy is accurate and preserves finest details, we can exploit variations in chromacity as well as luminance to enhance color images simply blending the processed luminance levels with the initial color. As recently observed [Gooch et al. 2005], the human visual system has better spatial acuity in luminance than in chrominance; by fusing the chrominance with co-located luminance changes, weak chroma variations are rendered sharper and better-localized. To the best of our knowledge we are the first to demonstrate such wide applicability of a grayscale operator. We obtain comparable results with specialized techniques for tasks like segmentation under different illuminants, detail enhancement and image dehazing (please refer to the supplemental video). Furthermore, we show that the approach is suitable for video decolorization, more challenging than images since the temporal coherence constraints may introduce flickering artifacts.

\section{References}

Gooch, A. A., Olsen, S. C., Tumblin, J., And Gooch, B. 2005. Color2gray: salience-preserving color removal. ACM Trans. Graph. 24, 3, 634-639.

Grundland, M., AND Dodgson, N. A. 2007. Decolorize: Fast, contrast enhancing, color to grayscale conversion. Pattern Recognition 40, 11, 2891-2896.

ItTI, L., Koch, C., AND Niebur, E. 1998. A model of saliency-based visual attention for rapid scene analysis. IEEE PAMI 2O(11), 1254-1259.

JudD, D. B. 1966. Fundamental studies of color vision from 1860 to 1960. Proc Natl Acad Sci U S A. 55, 6, 1313-1330.

Smith, K., Landes, P.-E., Thollot, J., And Myszkowski, K. 2008. Apparent greyscale. Computer Graphics Forum (EUROGRAPHICS) 27, 3, 193-200. 